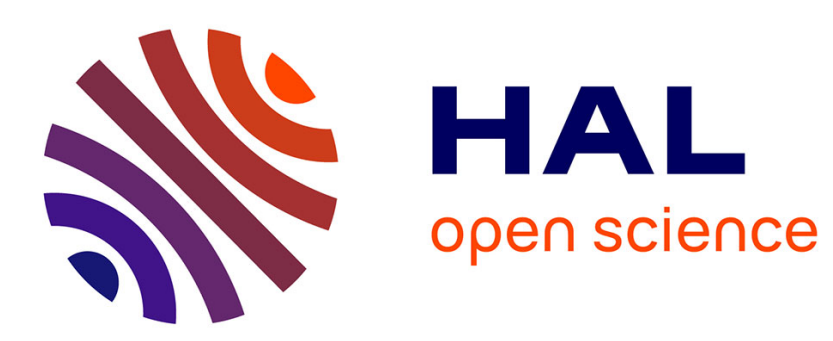

\title{
A Fast Computational Procedure to Solve the Multi-Item Single Machine Lot Scheduling Optimization Problem: the Average Cost Case
}

Laura S. Aragone, Roberto L.V. González

\section{- To cite this version:}

Laura S. Aragone, Roberto L.V. González. A Fast Computational Procedure to Solve the Multi-Item Single Machine Lot Scheduling Optimization Problem: the Average Cost Case. [Research Report] RR-2984, INRIA. 1996. inria-00073714

\section{HAL Id: inria-00073714 \\ https://hal.inria.fr/inria-00073714}

Submitted on 24 May 2006

HAL is a multi-disciplinary open access archive for the deposit and dissemination of scientific research documents, whether they are published or not. The documents may come from teaching and research institutions in France or abroad, or from public or private research centers.
L'archive ouverte pluridisciplinaire HAL, est destinée au dépôt et à la diffusion de documents scientifiques de niveau recherche, publiés ou non, émanant des établissements d'enseignement et de recherche français ou étrangers, des laboratoires publics ou privés. 


\section{A Fast Computational Procedure to Solve the Multi-Item Single Machine Lot Scheduling Optimization Problem : the Average Cost Case}

Laura S. Aragone - Roberto L.V. González

No 2984

Septembre 1996

THËME 4

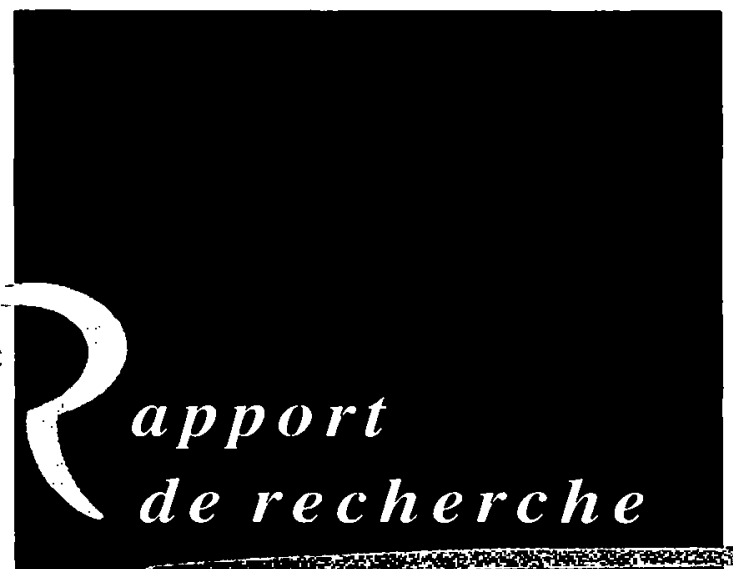


Les rapports de recherche de l'INRIA sont disponibles en format postscript sous ftp.inria.fr (192:93.2.54)

si vous n'avez pas d'accès ftp la forme papier peut être commandée par mail : e-mail:dif.gesdif@inria.fr (n'oublicz pas de mentionner votre adresse postale).
INRIA research reports are available in postscript format ftp.inria.fr (192.93.2.54)

if you haven't access by ftp we recommend ordering them by e-mail : e-mail : dif.gesdif@inria.fr (don't forget to mention your postal address).

par courrier : by mail :

\section{Centre de Diffusion Centre de Diffusion}

INRIA INRIA

BP 105 - 78153 Le Chesnay Cedex (FRANCE) BP.105 - 78153 Le Chesnay Cedex (FRANCE) 


\section{A FAST COMPUTATIONAL PROCEDURE TO SOLVE THE MULTI-ITEM SINGLE MACHINE LOT SCHEDULING OPTIMIZATION PROBLEM. THE AVERAGE COST CASE}

\section{UNE PROCEDURE NUMERIQUE POUR L'OPTIMISATION DE LA PRODUCTION D'UNE MACHINE MULTI-PRODUIT}

Laura S. Aragone * Roberto L.V. González *

* 'ONICL'T' - Dpto. Malemática, Fac. Cs. Ex., Ing. y Agr.. Universidad Nacional de Rusario - Avdal. Pellegrini 250 - 2000 Rosario - Argentine. This paper is inclucled in the activities developed in the framework of the Cooperation Project INRIA - Instituto de Matemática Beppo Levi, C'orordinators of the project: E. Rofman - R. (jonzález 


\begin{abstract}
In this pilper we present some especial procedures for the numerical solution of the optimal scheduling problem of a multi-item single machine. We study the infinite horizon (ase and the optimization criterion is the average cost. We establish the solution of the problem in terms of viscosity solutions of the Quasi-Variational Inequality (QVI) system associated to the problem. The existence of solution of the QVI and the uniquencss of the optimal arerage cost are proved. A method of discretization and a computational procedure are described which allows us to compute the solution in a short time and with precision of order $k$. We obtain an estimate for the discretization error and develop an algorithm that converges in a finite number of steps. In our method the nodes of the triangulation mesh are joined by segments of trajectories of the original system. This feature allows us to obtain the k-order precision which, in general, is impossible to obtain by usual methods.
\end{abstract}

\title{
Résumé
}

On présente ici quelques procédés spéciaux pour la solution numérique du problème optimal d'une machine multi-produit pour le cas d'horizon infini quand le critère d'optimisation est le coût moyen. On établit la solution du problème comme la soJution de viscosité d'un système d'inéquations quasi-variationnelles (QVI) associées au problème. On montre l'existence de solution du système QVI et l'unicité du coût optimal moyen. On donne une estimation pour l'erreur de discrétisation et on développe aussi un algoritlume très efficient qui converge dans un nombre fini de pas. On fait la description d'une méthode de discrétisation et d'un procédé permettant d'obtenir la solution en employant des petits temps de calcul avec un précision d'ordre $k, k$ étant la mesure de la discrétisation. La caractéristique principale de cette méthode est le fait que les nodes de la triangulation sont unis par des segments des trajectoires du système originel. Cette caractéristique permet d'obtenir la précision d'ordre $k$ qui, en général, est impossible d'obtenir par les méthodes habituelles.

Keywords: scheduling problems. quasi-uariational inequalities. Bellman equation. viscostity solution. antrage cost, numerrical solution.

Mots clefs: problimes dordonnacement. inéquations quasi-varialionnelles. équation de Bellman. solution de viscosité. cồt moyen. solution numérique.

AMS Classification: Primlary: 90B30, 90B35, 90B05, 90B 310

Secondary: $90(39,49 B 10,65 \mathrm{~K} 10$ 


\section{Contents}

1 Introduction . 1

2 Description of the problem $\quad 2$

2.1 Description of the production system . . . . . . . . . . . . 2

2.2 The set $\mathrm{Q}$ of admissible states . . . . . . . . . . . . . . . . . . 3

2.3 The evolution of the system . . . . . . . . . . . . . . . . 4

2.4 The set $A_{x}^{d}$ of admissible controls . . . . . . . . . . . . . 4

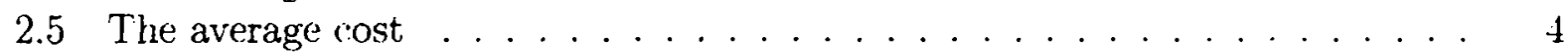

3 Use of viscosity techniques 5

3.1 Definition of the QVI system and its viscosity solution . . . . . . . . 5

3.2 Uniqueness property in terms of viseosity . . . . . . . . . . . 7

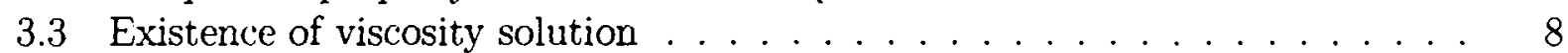

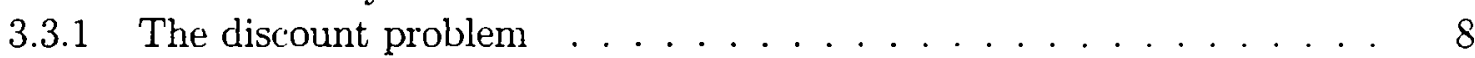

3.3.2 Relation between the two problems ............ 8

4 The discrete problem $\quad 11$

4.1 Elements of the discrete problem . . . . . . . . . . . . . . 11

4.1 .1 Approximation of domain $Q \ldots \ldots \ldots 11$

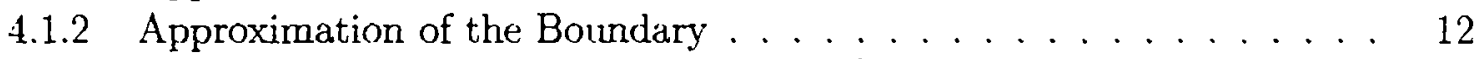

4.1.3 Definition of the approximation space $F^{k} \ldots \ldots \ldots 12$

4.1.4 Discretization of H-J-B inequalities . . . . . . . . . . . . 12

4.1 .5 Definition of operator $P^{k} \ldots \ldots \ldots \ldots \ldots$

4.1.6 Definition of the discrete problem . . . . . . . . . . . . . . 13

4.1 .7 Definition of operator $P_{k}^{\lambda} \ldots \ldots \ldots \ldots$

4.2 Relation between problems $\mathrm{P}_{k}$ and $\mathrm{P}_{k}^{\lambda} \ldots \ldots \ldots \ldots \ldots \ldots$

4.3 Convergence of the method . . . . . . . . . . . . . . . 19

5 Numerical algorithm 19

5.1 Preliminary definitions . . . . . . . . . . . . . . . . . . . . . . . . . . . . .

5.2 A fast algorithm . . . . . . . . . . . . . . . . . . . . . . . . . . . . . . . .

5.2.1 Definition of the aluxiliary function TEST . . . . . . . . . . 22

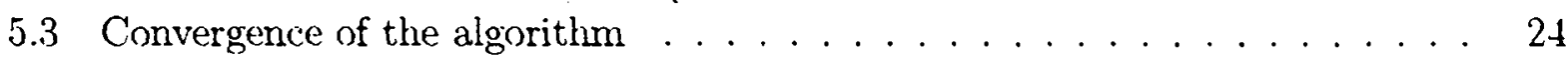

6 Applications $\quad 25$

7 Conclusions $\quad 26$ 


\section{List of Figures}

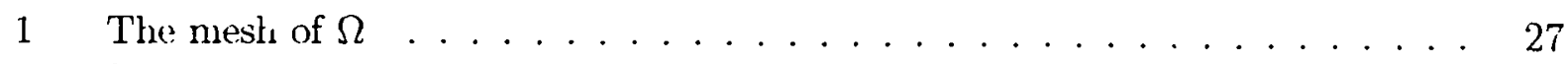

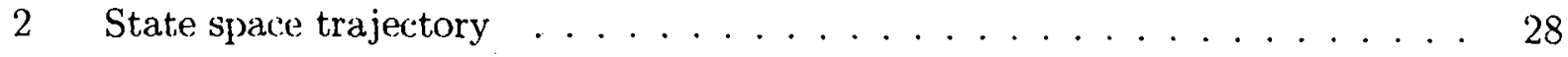




\section{Introduction}

In this paper we study the optimization of the production schedule of a multi-item single machine (see [2], [12], [13]). The objective is to find an optinial production schedule that minimizes the average cost for an infinite horizon. Speciffically we will try to minimize the following criterion

$$
J(\alpha(\cdot))=\limsup _{\nu \rightarrow \infty} \frac{1}{\theta_{\nu}} \sum_{i=1}^{\nu}\left(\int_{\theta_{\nu-1}}^{\theta_{\nu}} f\left(y(s), d_{i-1}\right) d s+q\left(d_{i-1}, d_{i}\right)\right)
$$

where $\theta_{i}$ are the switching times of the control policies used (see [1], [4], [8], [22], [24], [25], [26], [27], [28] and [29] for a more general description of similar problems).

By using dynamic programming techniques (see [9]) and taking into account the switching cost, it is possible to find an optimal feedback policy, in terms of any solution in the viscosity sense of the following first order Quasi-Variational Inequalities (QVI) system.

$$
\begin{cases}\frac{\partial U_{d}}{\partial x} g(d)+f-\mu \geq 0 & \text { in } \Omega \\ U_{d} \leq S^{d}(U) & \text { in } \Omega \\ \left(\frac{\partial U_{d}}{\partial x} g(d)+f-\mu\right)\left(U_{d}-S^{d}(U)\right)=0 & \text { in } \Omega\end{cases}
$$

being

$$
S^{d}(U)(x)=\min _{x \neq \tilde{x}}\left\{q(d, \tilde{d})+\dot{U_{\tilde{d}}}(x)\right\} \quad x \in Q, d \in D .
$$

This system is obtained considering a sequence of optinuzation problems with non zero discount rate. In a strict sense, the relation between the discount problem and the optimization with average cost problem is: $\forall x \in Q, \forall d \in D$

$$
\lim _{\lambda \rightarrow 0} \lambda U_{\lambda}=\mu=\inf _{\alpha(\cdot)} J(\alpha(\cdot))
$$

To obtain the numerical solution of system (2), we develop in this work a procedure based fundamentally in this convergence property, whose validity holds for the continuous problern as well as for the formulation associated to the fully discrete problem. We will obtain an estimation of the following type

$$
\left|\mu-\mu_{k}\right| \leq C k
$$

We propose an algorithm which converges in a finite number of steps. We also present rumerical results for the case $m=2$ (optimization of a machine with two items). 


\section{Description of the problem}

\subsection{Description of the production system}

At any time the machine is either idle or producing any one of $m$ different items. We will denote by

- $d=0$ the idle state of the machine

- $d=1, \ldots, m$, when it is producing item $d$.

For each item $d=1, \ldots, m$; we define the problem data as follows

- $r_{d}$ the demand by unit time of item $d$

- $p_{d}$ the production quantity by unit time at the machine setting $d$

- $M_{d}$ the inventory capacity constraint of item $d$

- $q(d, \tilde{d})$ the switching cost of the machine from state $d$ to $\tilde{d}$

- $f(x, d)$ the instantaneous inventory-holding/production cost.

We will always assume a non zero loop cost condition:

$\exists q_{0}>0$ such that for any closed loop $d_{0}, d_{1}, \ldots, d_{p}, d_{p-1}$, with $d_{0}=d_{p-1}, p \leq m$, we have

$$
\sum_{i=0}^{p} q\left(d_{i}, d_{i-1}\right) \geq q_{0}
$$

and we suppose that the following conditions are veritied

$$
\begin{aligned}
& q(d, \tilde{d}) \geq 0 \quad \forall \tilde{d} \neq d . \quad q(d, d)=0 \quad \forall d \in D, \\
& q(d, \bar{d}) \leq q(d, \widetilde{d})+q(\bar{d}, \bar{d}), \quad \forall d \neq \tilde{d} \neq \bar{d} .
\end{aligned}
$$

In addition. we assume that the switching time is small enough to be disregarded and that the following condition, under which a feasible schedule exist. holds

$$
\sum_{d=1}^{m} \frac{r_{d}}{p_{d}}<1
$$

In fart, we will always assume $(6)$, because condition $\sum_{d=1}^{m} \frac{r_{d}}{p_{d}}=1$, forbids the machine to be in the idle state except for a total time $\tau=\sum_{d=1}^{m} \frac{x_{d}}{p_{d}}$, and this is not a natural condition for a problem with infinite horizon. 


\subsection{The set $Q$ of admissible states}

Let $y_{d}(t)$ be the inventory level of item $d$ at time $t$, starting at $y_{d}(0)=x_{d}$. Therrfore, for the global state " $y$ " of the system, we have

$$
\begin{gathered}
y(t)=\left(y_{1}(t), \ldots, y_{m}(t)\right) \\
\left(y_{1}(0), \ldots, y_{m}(0)\right)=\left(x_{1}, \ldots, x_{m}\right) .
\end{gathered}
$$

As neither backlogging nor production over the capacity constraints are allowed for the inventory state $y_{d}$, the following restriction holds

$$
0 \leq y_{d} \leq M_{d}, \quad \forall d=1, \ldots, m
$$

Let us divide the $x_{i}$ values into three categories

$$
\mid \begin{aligned}
& x_{i}=0 \\
& 0<x_{i}<M_{i} \\
& x_{i}=M_{i}
\end{aligned}
$$

An $x$ point is classified using an m-tuple of digits $a(x)=\left(a_{1}, \ldots, a_{m}\right)$, where

$$
\mid \begin{array}{lll}
x_{i}=0 & \Rightarrow & a_{i}=0 \\
0<x_{i}<M_{i} & \Rightarrow & a_{i}=1 \\
x_{i}=M_{i} . & \Rightarrow & a_{i}=2 .
\end{array}
$$

Let us refine

$$
\Gamma\left(a_{1}, \ldots, a_{m}\right) \equiv\left\{x: a(x)=\left(a_{1}, \ldots, a_{m}\right)\right\}
$$

The set $Q$ of admissible states comprises only the set of points with at most one zero component, because if we start from other points that do not verify this condition. we cannot avoid the shortage of at least one item, i.e.

$$
Q=\bigcup_{a}\left\{\Gamma\left(a_{1}, \ldots, a_{i}, \ldots, a_{m}\right): \text { at most one component } a_{i}=0\right\}
$$

Let us denote with $\partial Q^{-}$the points of $Q$ that are not admissible, i.e.

$$
\partial Q^{-}=\bigcup_{a}\left\{\Gamma\left(a_{1}, \ldots, a_{i}, \ldots, a_{m}\right): \text { at least two } a_{i}=0\right\} .
$$

If we denote with $\Omega$ the interior of $Q$, we have

$$
\Omega \equiv\left\{x: 0<x_{i}<M_{i}, i=1, \ldots, m\right\}=\Gamma(1, \ldots, 1) .
$$




\subsection{The evolution of the system}

For any step function $\alpha:[0, \infty) \rightarrow D$ from the definition of $r_{d}, p_{d}$, the following equation of evolution holds

where

$$
\frac{d y}{d t}=g(\alpha(t))
$$

being

$$
g(\alpha)=\left(g_{1}(\alpha), \ldots, g_{m}(\alpha)\right)
$$

$$
g_{d}(\alpha)=\mid \begin{array}{ll}
-r_{d} & \text { if } \alpha \neq d \\
p_{d}-r_{d} & \text { if } \alpha=d .
\end{array}
$$

Remark 2.1 Since $g$ is piece-wise constant, the equation (13) has global solution for any control policy. At the same time we always suppose that the function $f$ is uniformly Lipschitz in $Q, \forall d \in D$.

\subsection{The set $A_{x}^{d}$ of admissible controls}

An admissible schedule is characterized by a sequence of pairs $\left\{\theta_{i}, d_{i}\right\}$, where $\theta_{i}$ is the switching time.

$$
0 \leq \theta_{0} \leq \theta_{1}<\ldots<\theta_{i}<\theta_{i+1}<\ldots
$$

and $d_{i} \in D: d_{i} \neq d_{i-1} ; i=0,1, \ldots$ is the state of production in $\left(\theta_{i}, \theta_{i-1}\right]$.

For each $x \in Q, d \in D$. we denote $A_{x}^{d}$ the set of all admissible schedules with initial state $x$ and initial machine setting $d$

$$
A_{x}^{d}=\left\{\alpha(\cdot)=\left(\theta_{i}, d_{i}\right)_{i=0}^{\infty}: d_{0}=d, \forall t \in \Re \cdots, y(t) \in Q\right\}
$$

In other words, we will consider sequences $\left\{\theta_{i}, d_{i}\right\}$ such that the associated trajectories remain in $Q, \forall t \geq 0$.

\subsection{The average cost}

To cach control policy $\alpha(\cdot)$ we associate the cost function (1). For each $d \in D$ and $x \in Q$, we define the minimum average cost.

$$
\mu_{d}(x)=\inf \left\{J(\alpha(\cdot)): \alpha(\cdot) \in A_{x}^{d}\right\} .
$$

Our objective is to find $\forall x \in Q$ and $\forall d \in D$, a policy $\tilde{\alpha}_{x}^{d}(\cdot) \in A_{x}^{d}$. such that

$$
J\left(\bar{\alpha}_{x}^{d}(\cdot)\right)=\mu_{d}(x) \text {. }
$$

In the following proposition we will see that $\mu_{d}(x)$ does not depend of $d$ and $x$.

Proposition $2.1 \exists \mu \in \Re$ such that

$$
\mu_{d}(x)=\mu \quad \forall x \in Q, \forall d \in D .
$$


Proof. Let $\hat{x}, x \in Q$ and $\hat{d} d \in D$. Taking into account the hypotheses about the dynamic of the system, it is inmediate to prove, by using the same techniques as those employed in [13], that there exists a policy $\alpha(\cdot) \in A_{x}^{d}$ and $\hat{t} \geq 0$, such that

$$
\alpha(\hat{t})=\hat{x}, \quad y_{x}(\hat{t})=\hat{d}
$$

Let $\hat{\alpha} \in A_{\tilde{x}}^{\dot{\alpha}}$, we define $\alpha_{1} \in A_{x}^{d}$ such that

$$
\alpha_{1}(t)=\mid \begin{array}{ll}
\alpha(t) & 0 \leq t \leq \hat{t}, \\
\hat{\alpha}(t-\hat{t}) & t>\hat{t} .
\end{array}
$$

In consequence

$$
J\left(\alpha_{1}(\cdot)\right)=J(\hat{\alpha}(\cdot))
$$

From the definition (17)

$$
\mu_{d}(x) \leq J\left(\alpha_{1}(\cdot)\right)
$$

then, as the policy $\hat{\alpha}(\cdot)$ is an arbitrary admissible one, it holds

$$
\mu_{d}(x) \leq \mu_{\tilde{d}}(\hat{x})
$$

As $(x, d)$ and $(\hat{x}, \hat{d})$ are also arbitrary, we conclude

$$
\mu_{\hat{d}}(x)=\mu_{d}(x)=\mu .
$$

\section{Use of viscosity techniques}

We will use viscosity techniques (see [7]), in order to consider general solutions of the Hamilton-Jacobi-Belman (H-J-B) equations system associated to this problem. Also with these techniques, we can easily prove properties of uniqueness of solution and convergence results.

\subsection{Definition of the QVI system and its viscosity solution}

Let us define

$$
\partial Q_{e}=\bigcup_{i=1}^{m}\left(\gamma_{i}^{+} \bigcup \gamma_{i}^{-}\right)
$$

where

$$
\begin{aligned}
& \gamma_{d}^{+}=\bigcup_{a}\left\{\Gamma\left(a_{1}, \ldots, a_{d}, \ldots, a_{m}\right): a_{d}=2\right\} \cap Q, \\
& \gamma_{d}^{-}=\bigcup_{a}\left\{\Gamma\left(a_{1}, \ldots, a_{d}, \ldots, a_{m}\right): a_{d}=0\right\} \bigcap Q
\end{aligned}
$$


and

$$
\partial Q_{e}=\bigcup_{a}\left\{\Gamma\left(a_{1}, \ldots, a_{d}, \ldots, a_{m}\right): \text { at least two coefficients } a_{d}=0\right\}
$$

By using the same methodology as that employed in [15], [16], we will say that, $\left(U_{d}, \mu\right)$ are a viscosity solution of (2), with boundary conditions (23)-(25) if

- they are continuous functions in $Q$

- they satisfy $U_{d} \leq S^{d}(U)$

- they verify the following boundary conditions, for $x \in\left(\partial Q_{e} \cup \partial Q^{r}\right)$

$$
\begin{gathered}
U_{\dot{d}}(x)=S^{\dot{d}}(U)(x) \quad \forall \tilde{d} \neq d \quad \text { if } x \in \gamma_{d}^{-} \\
U_{d}(x)=S^{d}(U)(x) \quad \text { if } x \in \gamma_{d}^{--} \\
\lim _{x \rightarrow \partial Q^{+}} U_{d}(x)=+\infty
\end{gathered}
$$

- $\frac{\partial U_{d}}{\partial x} g(d)+f-\mu \geq 0$ in $\Omega$ in the viscosity sense, i.e.

$\forall \dot{\psi} \in C^{1}(\Omega)$, if $U_{d}-\psi$ has a local maximum in $x_{0}$, then

$$
\frac{\partial \psi}{\partial x}\left(x_{0}\right) g(d)+f\left(x_{0}, d\right)-\mu \geq 0
$$

- $\forall x \in \Omega / U_{d}(x)<S^{d}(U)(x)$, then $\exists \delta(x)$ such that

$$
\frac{\partial U_{d}}{\partial x} g(d)+f-\mu=0 \quad \text { in } B_{\delta(x)} \text { in the viscosity sense, i.e. }
$$

$\forall \psi \in C^{1}\left(B_{\delta(x)}(x)\right), \quad$ if $U_{d}-\psi$ has a local maximum in $x_{0}$, then

$$
\frac{\partial \psi}{\partial x}\left(x_{0}\right) g(d)+f\left(x_{0}, d\right)-\mu \geq 0
$$

$\forall \psi \in C^{\mathrm{l}}\left(B_{\delta(x)}(x)\right), \quad$ if $U_{d}-\psi$ has a local minimum in $x_{0}$, then

$$
\frac{\partial \psi}{\partial x}\left(x_{0}\right) g(d)+f\left(x_{0}, d\right)-\mu \leq 0 .
$$




\subsection{Uniqueness property in terms of viscosity}

\section{A constructive procedure for an optimal policy}

Let $U_{d}$ be any set of continuous functions, which are solutions in the viscosity sense of the QVI system (2), with boundary conditions (23)-(25). By using them, an optimal feedback policy $\alpha^{*}=\left\{\theta_{i}, d_{i}\right\} \in A_{x}^{d}$ can be obtained in the following way:

We define

$$
\theta_{0}=0, \quad d_{0}=d
$$

and recursively

$$
\begin{gathered}
\theta_{i}=\min \left\{t \geq \theta_{i-1}: U_{d_{i-1}}(y(t))=\left(S^{d_{i-1}}(U)\right)(y(t))\right\} \\
d_{i} \in\left\{d \in D:\left(S^{d_{i-1}}(U)\right)\left(y\left(\theta_{i}\right)\right)=q\left(d_{i-1}, d_{i}\right)+U_{d}\left(y\left(\theta_{i}\right)\right), d_{i-1} \neq d_{i}\right\} .
\end{gathered}
$$

Next theorem establishes the optinality of the procedure.

Theorema 3.1 If $U$ is a continuous viscosity solution of the system (2), (2.3)-(25), then the policy constructed according to (26)-(27), satisfies

$$
J\left(\alpha^{*}(\cdot)\right)=\inf \left\{J(\alpha(\cdot)): \alpha \in A_{x}^{d}\right\} .
$$

The proof uses essentially the procedure employed in [13], [16] and it will not be included here for the sake of briefness.

Corolary 3.1 There exists at most one value of the parameter $\mu$ such that (2), (23)-(25) has a solution in the viscosity sense.

Proof. Let us suppose that $\left(U^{1}, \mu_{1}\right)$, and $\left(U^{2}, \mu_{2}\right)$ are two solutions in the sense of viscosity of $(2),(23)-(25)$.

It can be seen, by using the same techriques employed in [16], that in terms of a solution $U^{\prime}$ we can find $\bar{\alpha}_{i}(\cdot)$ such that

$$
\mu_{i}=J\left(\bar{\alpha}_{i}(\cdot)\right)
$$

and $\forall \alpha \in A_{x}^{d}$, it is verified

$$
J(\alpha(\cdot)) \geq \mu_{i}
$$

In consequence

$$
\mu_{1} \leq J\left(\bar{\alpha}_{2}(\cdot)\right)=\mu_{2} \leq J\left(\bar{\alpha}_{1}(\cdot)\right)=\mu_{1}
$$

Remark 3.1 If $U$ is a solution of (2), (23)-(25), then $U+c \cdot e$, is also a solution $\forall c \in \Re$, bcing $e=(1, \ldots, 1) \in \Re^{m+1}$. 


\subsection{Existence of viscosity solution}

\subsubsection{The discount problem}

A Lipschitz-continuous solution of the system (2) with boundary conditions (23)-(25) can be obtained considering a sequence of optimization problems with non zero discount rate (we denote $\lambda$ this coefficient). For this type of problem, the solution is given by the unique solution in the viscosity sense of the (QVI) system (28), with the boundary conditions (23)(25).

$$
\begin{cases}\frac{\partial U_{d}^{\lambda}}{\partial x} g(d)+f-\lambda U_{d}^{\lambda} \geq 0 & \text { in } \Omega \\ U_{d}^{\lambda} \leq S^{d}\left(U^{\lambda}\right) & \text { in } \Omega \\ \left(\frac{\partial U_{d}^{\lambda}}{\partial x} g(d)+f-\lambda U_{d}^{\lambda}\right)\left(U_{d}^{\lambda}-S^{d}\left(U^{\lambda}\right)\right)=0 & \text { in } \Omega .\end{cases}
$$

The viscosity solution is defined in the same form as that used for the system (2), (23)-(25): (see [2], [15], [16]).

\subsubsection{Relation between the two problems}

The relation between the discount problem and the optinal average problen is given by the following theorem. It gives a strict statement of the intuitive fact that problems with a low discount rate or with average cost are optimized by similar policies.

Theorema 3.2 By virtue of the feasibility condition (6), the following properties hold.

$$
\begin{aligned}
& \lim _{\lambda \rightarrow 0} \lambda U_{d}^{\lambda}=\mu \quad \forall x \in Q, \forall d \in D \\
& \forall U \in\left(\bigcap_{\varsigma>0}\left(\overline{\bigcup_{\varsigma>\lambda>0}\left(U^{\lambda}(\cdot)-U_{d_{n}}^{\lambda}\left(x^{0}\right) \cdot e\right)}\right)\right), \\
& (U, \mu) \text { is solution of }(2),(23)-(25) .
\end{aligned}
$$

Remark 3.2 In (29) and in the following proof we consider the topology of uniform convergence in $C(Q)$.

Proof. As we have seen in the previous section, the following estimation holds

$$
0 \leq U_{d}^{\lambda} \leq C\left(1+\frac{1}{\lambda}+\left(\log \left(d\left(x, \partial Q^{-}\right)\right)\right)^{-}\right), \quad \forall x \in Q, \forall d \in D,
$$

in consequence for $x \in Q, d \in D$, it restuts

$$
\left|\lambda U_{d}^{\lambda}(x)\right| \leq C\left(1+\lambda\left(1+\left(\log \left(d\left(x, \partial Q^{-}\right)\right)\right)^{-}\right)\right) .
$$


Moreover, when $K^{\prime}$ is a compact subset of $Q$, the functions $U^{\lambda}$ are uniformly Lipschit: continuous, being the Lipschitz constiant $L\left(K^{\prime}\right)$ independent of $\lambda$. i.e. we have

$$
\left\|U_{d}^{\lambda}(\cdot)-U_{d_{0}}^{\lambda}\left(x^{0}\right)\right\|_{W^{1, \infty}(K)} \leq L\left(K^{-}\right)
$$

Let

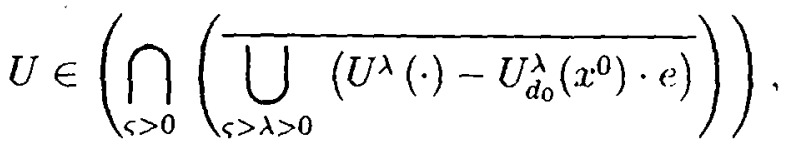

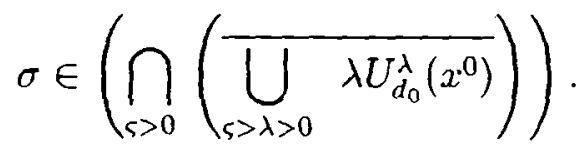

Then, there exists a sub-sequence $\lambda_{v} \rightarrow 0$ (that, in order to alleviate the notation. we will denote $\lambda$ ), with the following properties

$$
\begin{gathered}
U_{d}^{\lambda}(\cdot)-U_{d_{0}}^{\lambda}\left(x^{0}\right) \rightarrow U_{d}(\cdot) . \\
\lambda U_{d_{0}}^{\lambda}\left(x^{0}\right) \rightarrow \sigma .
\end{gathered}
$$

We should remark that (31) implies

$$
\lambda U_{d}^{\lambda}(x) \rightarrow \sigma \quad \forall(x, d) \in Q \times D .
$$

Let us see that $(U, \sigma)$ is a solution in the viscosity sense of (2). (23)-(25). By virtue of the uniform convergence in (32), the following properties are verified

- $U_{d}$ are continuous functions in $Q$

- $U_{d}$ satisfies $U_{d} \leq S^{d}(U)$

- $U_{d}$ satisfies (23)-(25)

- $\frac{\partial U_{d}}{\partial x} g(d)+f-\sigma \geq 0$ in $\Omega$ in the riscosity sense; in fact.

let $\psi \in C^{1}(\Omega)$ such that

$$
\left(U_{d}-\psi\right)(\tilde{x})=0>\left(U_{d}-\psi\right)(x) \quad \forall x \in N(\tilde{x}) \backslash\{\tilde{x}\}
$$

(where we denote $N(\tilde{x})$ a neighborhood of point $\tilde{x} \in \Omega$ ).

For each $\lambda$, let $x^{\lambda} \in N(\tilde{x})$ such that it is a local maximum of $\left(U_{d}-\psi\right)$. i.e.

$$
\left(U_{d}^{\lambda}-\psi\right)\left(x^{\lambda}\right) \geq\left(U_{d}^{\lambda}-\psi\right)(x) \quad \forall x \in N(\tilde{x}) .
$$

Since $\tilde{x}$ is a strict maximum of $U_{d}-\psi$ in $N(\tilde{x})$, and as we are working with a uniformly convergent sub-sequence of $\left\{U^{\lambda}(\cdot)-U_{d_{0}}^{\lambda}\left(x^{0}\right)\right\}$, we have

$$
x^{\lambda} \rightarrow \tilde{x} \text {. }
$$


Then, as $U^{\lambda}$ is a viscosity solution of (28), it holds

$$
\frac{\partial \psi}{\partial x}\left(x^{\lambda}\right) g(d)+f\left(x^{\lambda}, d\right)-\lambda U_{d}^{\lambda}\left(x^{\lambda}\right) \geq 0
$$

by taking limit as $\lambda \rightarrow 0$, it results

$$
\frac{\partial \psi}{\partial x}(\tilde{x}) g(d)+f(\tilde{x}, d)-\sigma \geq 0
$$

- $\forall \tilde{x} \in \Omega / U_{d}(\tilde{x}) \leq S^{d}(U)(\tilde{x})$, then $\exists \delta(\tilde{x})$ such that

$$
\frac{\partial U_{d}}{\partial x} g(d)+f-\sigma=0 \quad \text { in } B_{\delta(\tilde{x})}(\tilde{x}) \text { in the viscosity sense. }
$$

Let $\psi \in C^{1}\left(B_{\delta(\tilde{x})}(\tilde{x})\right)$, such that

$$
\left(U_{d}-\psi\right)(\bar{x})>\left(U_{d}-\psi\right)(x) \quad \forall x \in B_{\delta(\dot{x})}(\tilde{x}) \backslash\{\tilde{x}\} .
$$

In a similar way it is proved

$$
\frac{\partial \psi}{\partial x}(\bar{x}) g(d)+f(\bar{x}, d)-\sigma \geq 0
$$

To obtain the other inequality, let $\psi \in C^{1}\left(B_{\delta(\tilde{x})}(\tilde{x})\right)$. such that

$$
\left(U_{d}-\psi\right)(\bar{x})<\left(U_{d}-\psi\right)(x) \quad \forall x \in B_{\delta(\tilde{x})}(\tilde{x}) \backslash\{\tilde{x}\} .
$$

As a consequence of both the uniform convergence of $\left\{U^{\lambda}(\cdot)-U_{d_{0}}^{\lambda}\left(x^{0}\right)\right\}$ and the continuity of operator $S$, for $\lambda$ large enough it results

$$
U_{d}^{\lambda} \leq S^{d}\left(U^{\lambda}\right) \quad \text { in } B_{\delta(\tilde{x})}(\tilde{x}) .
$$

For each $\lambda$ let $x^{\lambda} \in B_{\delta(\tilde{x})}(\tilde{x})$, such that

$$
\left(U_{d}^{\lambda}-\psi^{\prime}\right)\left(x^{\lambda}\right) \leq\left(U_{d}^{\lambda}-\psi\right)(x) \quad \forall x \in B_{\delta(\tilde{x})}(\tilde{x}) .
$$

Then, by definition of viscosity solution of the system (28), it results

$$
\frac{\partial \psi}{\partial x}\left(x^{\lambda}\right) g(d)+f\left(x^{\lambda}, d\right)-\lambda U_{d}^{\lambda}\left(x^{\lambda}\right) \leq 0
$$

Clearly, as $x^{\lambda} \rightarrow \bar{x}$, by taking limit as $\lambda \rightarrow 0$, it results

$$
\frac{\partial \psi}{\partial x}(\vec{x}) g(d)+f(\bar{x}, d)-\sigma \leq 0
$$

Then $(U, \sigma)$ is a solution in the sense of viscosity of (2). By virtue of proposition 2.1, it results $\sigma=\mu$ and in consequence every sequence $\lambda U^{\lambda}$ converges to $\mu$. 


\section{The discrete problem}

\subsection{Elements of the discrete problem}

To define the discrete problem, we introduce an approximation which comprises a discretization of the space $W_{\text {luc }}^{1, \infty}(\Omega)$ and a discretization of conditions (23)-(24). We use the techniques analyzed in [3], [5], \{14], \{18], [19], [20] and the notations employed in [2].

\subsubsection{Approximation of domain $Q$}

We will approximate $Q$ with $Q_{k}=\bigcup_{j} S_{j}^{k}$, where $S_{j}^{k}$ is a finite set of quadrilateral elements and, in consequence, $Q_{k}$ is a polyhedron of $\Re^{m}$. We define

$$
k=\max _{j}\left(\operatorname{diam}\left(S_{j}^{k}\right)\right) \text {. }
$$

We use a special uniform mesh $B^{k}$ of the space $\Re^{m}$. This mesh is defined in terms of an arbitrary parameter $h$, in the following way

$$
\begin{aligned}
& B^{k}=\left\{x^{0}+\sum_{d=0}^{m} \varsigma_{d} e^{d}: \varsigma_{d} \text { integer }\right\} \\
& h_{d}=\frac{r_{d}}{p_{d}} h \\
& h_{0}=\left(1-\sum_{d=1}^{m} \frac{r_{d}}{p_{d}}\right) h \\
& e^{0}=\left(-r_{1}, \ldots,-r_{i}, \ldots,-r_{m}\right) h_{0} \\
& e^{d}=\left(-r_{1}, \ldots,-r_{d-1}, p_{d}-r_{d},-r_{d-1}, \ldots,-r_{m}\right) h_{d}
\end{aligned}
$$

We will say that $S_{j}^{k}$ is an elementary domain of $Q_{k}$ if it has the following form

$$
S_{j}^{k}=x^{k}+\left\{x=\sum_{d=0}^{m} \varsigma_{d} e^{d}: \varsigma_{d} \in[0,1]\right\}, \quad x^{k} \in B^{k}, \quad S_{j}^{k} \subset Q .
$$

We will denote with $V^{k}=\left\{x^{i}, i=1, \ldots N\right\}$ the set of nodes of $Q_{k}$ and we will denote the cardinal of $V^{k}$ by $N$. The typical shape of this mesh can be seen in Figure 1.

Remark 4.1 If $k$ is small enough, for any two vertices of $V^{k}$, there always exists a path given by a natural trajectory of the system that joins the first one with the second one.

Remark 4.2 From (6) and (34) it results that $B^{k}$ can be generated by

$$
B^{k}=\left\{x^{0}+\sum_{d=1}^{m} \varsigma_{d} e^{d}: \varsigma_{d} \text { is an integer }\right\} .
$$


Definition 4.1 Discrete controls associated to the mesh.

We introduce a special family of controls by restricting the distance between switching tinues in the following way

$$
A_{x}^{d, k}=\left\{\alpha(\cdot) \in A_{x}^{d}: \theta_{i+1}-\theta_{i}=\varsigma h^{d_{i}}, \quad \zeta \text { is an integer }\right\} .
$$

\section{An Interpretation of the Mesh}

The special mesh we use originates a discrete optimal control problem. In that problem, the system has an evolution given by the differential equation (13). but controls $d_{i}$ are applied during intervals whose length is $\varsigma h^{d_{i}}$ and the initial state $x$ must be a node of $V^{k}$.

In consequence, the trajectory associated to this control reaches a node of the mesh at every switching time.

Taking into account the interpretation of the discrete equations as the optimality conditions over the Markov chain associated to this discretization, this interpretation implics that the chain is deterministic in the sense that $P_{i, j}=0$ or 1 , etc. (see [23]).

This property of the mesh plays a key role in relation to the precision of the method and the velocity of convergence of its computational algorithm.

\subsubsection{Approximation of the Boundary}

We define, $\forall d=1 \ldots . m$.

$$
\begin{aligned}
& \gamma_{k, d}^{-}=\left\{x^{i} \in V^{k}: x^{i}+h^{d} g(d) \notin Q_{k}\right\} . \\
& \hat{\gamma_{k, d}^{-}}=\left\{x^{i} \in V^{k}: x^{i}+h^{\hat{d}} g(\hat{d}) \notin Q_{k}, \forall \hat{d} \neq d\right\} .
\end{aligned}
$$

\subsubsection{Definition of the approximation space $F^{k}$}

We consider the set $F^{k}$ of functions $w: Q^{k} \times D \rightarrow \Re, w(\cdot, d) \in W^{1, \infty}\left(Q_{k}\right)$, such that in each quadrilateral element $\mathrm{Q}_{k}, w(\cdot, d)$ is a polynomial which belongs to the $\mathrm{Q}^{1}$ family (see [6], [10], [11] for the corresponding definitions). It is obvious that any $w \in F^{k}$ is uniquely characterized by the values $w\left(x^{i}, d\right), x^{i} \in V^{k}, d \in D$.

\subsubsection{Discretization of $\mathrm{H}-\mathrm{J}-\mathrm{B}$ inequalities}

We will use the following discretization of conditions (2)

$$
\begin{cases}w\left(x^{i}, d\right) \leq D_{d}^{k}\left(w, \mu^{k}\right)\left(x^{i}\right) & \forall\left(x^{i}, d\right) \in V^{k} \times D \\ u\left(x^{i}, d\right) \leq S^{\prime d}(u)\left(x^{i}\right) & \forall\left(x^{i}, d\right) \in V^{k} \times D .\end{cases}
$$


We define $D_{d}^{k}\left(u \cdot \mu^{k}\right)\left(x^{i}\right)$ in the following form

$$
\begin{aligned}
& \left(D_{d}^{k}\left(w, \mu^{k}\right)\right)\left(x^{i}\right)=w\left(x^{i}+h^{d} g(d), d\right)+\int_{0}^{h_{d}} h^{l}\left(f\left(x^{i}+s g(d), d\right)-\mu^{k}\right) d s
\end{aligned}
$$

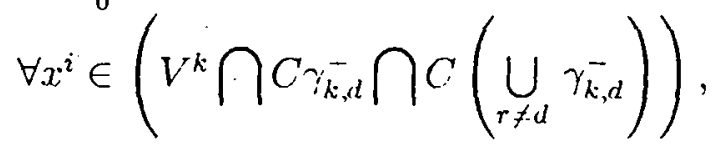

$$
\begin{aligned}
& \left(D_{d}^{k}\left(w, \mu^{k}\right)\right)\left(x^{i}\right)=+\infty \quad \forall x^{i} \in\left(\underset{\gamma_{k, d}^{+} \bigcup}{\bigcup}\left(\bigcup_{r \neq i d} \gamma_{k, d}^{-}\right)\right) .
\end{aligned}
$$

Remark 4.3 We can see that the definition of $D_{d}^{k}$ is consistent with (2) and takes into account the constraints (23)-(25).

Remark 4.4 We can use (41) instead of (40):

$$
\begin{array}{r}
\left(D_{d}^{k}\left(w, \mu^{k}\right)\right)\left(x^{i}\right)=w\left(x^{i}+h^{d} g(d) \cdot d\right)+h^{d}\left(f\left(x^{i}, d\right)-\mu^{k}\right) \\
\forall x^{i} \in\left(V^{k} \bigcap C \gamma_{k, d}^{-} \cap C\left(\bigcup_{r \neq d} \gamma_{k, d}^{-}\right)\right) .
\end{array}
$$

\subsubsection{Definition of operator $P^{k}$}

We define the operator $P_{k}: F_{k} \times \Re \rightarrow F_{k}$ in the following form

$$
P_{k}\left(w, \mu^{k}\right)\left(x^{i}, d\right)=\min \left(\left(D_{d}^{k}\left(w, \mu^{k}\right)\right), S^{d}(w)\left(x^{i}\right)\right) . \quad \forall\left(x^{i}, d\right) \in V^{k} \times D
$$

\subsubsection{Definition of the discrete problem}

In relation to the QVI system, we introduce the following problem which is defined in a similar way to that we employed in [2].

$$
\text { Problem } \mathrm{P}_{k}: \text { Find }\left(w, \mu^{k}\right) \text { such that } w=P_{k}\left(w \cdot \mu^{k}\right)
$$

\section{Definitions of discrete policies and auxiliary concepts}

- Set of multi-valued or generalized discrete policies

$$
\Lambda=\left\{A: V^{k} \times D \rightarrow P(D)\right\} .
$$

- Set of mono-valued discrete policies

$$
\Theta=\left\{A \in \Lambda: \forall\left(x^{i}, d\right) \in V^{k} \times D, \operatorname{card}\left(A\left(x^{i}, d\right)\right)=1\right\} .
$$


- Transitions associated to policies

Let $A \in \Theta$. We define

$$
T\left(x^{i}, d\right)= \begin{cases}T\left(x^{i}, d\right)=x^{i} & \text { if } d \neq A\left(x^{i}, d\right), \\ T\left(x^{i}, d\right)=x^{i}+h^{d} g(d) . & \text { if } d=A\left(x^{i}, d\right) .\end{cases}
$$

- Cost of elemental transitions

Let $w \in F_{k}, x^{j} \in V^{k}$ and a policy $A \in \Theta$

$$
F_{d}\left(x^{j}, A\right)= \begin{cases}q\left(d, A\left(x^{j}, d\right)\right) & \text { if } d \neq A\left(x^{j}, d\right) \\ h^{d} f_{d}\left(A\left(x^{j}, d\right)\right) & \text { if } d=A\left(x^{j}, d\right) .\end{cases}
$$

- Cycles associated to a policy

Let a discrete policy $A \in \Theta$, we will say that

$$
C=\left\{\left(x^{1}, d_{1}\right), \ldots,\left(x^{q}, d_{q}\right)\right\}
$$

is one of the cycles associated to that policy if

$$
\left\{\begin{array}{l}
\left(x^{j \div 1}, d_{j+1}\right)=\left(T\left(x^{j}, d_{j}\right), A\left(x^{j}, d_{j}\right)\right) \quad \forall j=1, \ldots, q \\
\left(x^{1}, d_{1}\right)=\left(T\left(x^{q}, d_{q}\right), A\left(x^{q}, d_{q}\right)\right)
\end{array}\right.
$$

- Average costs associated to a policy

Each cycle $C$ previously defined has the following associated average cost

$$
\mu(C)=\frac{\sum_{j=1}^{q} F_{d}\left(x^{j}, A\right)}{\sum_{j=1, d_{j} \neq 0}^{q}\left(\frac{\left(x^{j-1}\right)_{d_{j}}-\left(x^{j}\right)_{d_{j}}}{p_{d_{j}}-r_{d_{j}}}\right)+\sum_{j=1, d_{j}=0}^{q}\left(\frac{\left(x^{j+1}\right)_{1}-\left(x^{j}\right)_{1}}{r_{1}}\right)}
$$

Remark 4.5 For the $j$ index we use the addition modulus $q$, i.e. $x^{q+1}=x^{1}$.

Remark 4.6 To unify notation we define

$$
\left(\frac{\left(x^{j+1}\right)_{d_{j}}-\left(x^{j}\right)_{d_{j}}}{p_{d_{j}}-r_{d_{j}}}\right)=\left(\frac{\left(x^{j+1}\right)_{1}-\left(x^{j}\right)_{1}}{r_{1}}\right) \quad \text { for } d_{j}=0
$$


in eonsequeree we obtain the following notation

$$
\mu(C)=\frac{\sum_{j=1}^{q} F_{d}\left(x^{j}, A\right)}{\sum_{j=1}^{q}\left(\frac{\left(x^{j+1}\right)_{d_{j}}-\left(x^{j}\right)_{d_{j}}}{p_{d_{j}}-r_{d_{j}}}\right)}
$$

- Optimal discrete control associated to $(w, \mu): \Phi$

$$
\begin{aligned}
& \Phi(w, \mu) \subset \Lambda \\
& \Phi(w, \mu)\left(x^{i}, d\right)=\left\{\tilde{d}: P_{k}(w, \mu)\left(x^{i}, d\right)=w\left(x^{i}, \tilde{d}\right)+q(d, \tilde{d})\right\} \bigcup B\left(x^{i}, d\right) .
\end{aligned}
$$

where

$$
B\left(x^{i}, d\right)=\mid \begin{array}{ll}
\{d\} \quad \text { if } P_{k}(w, \mu)\left(x^{i}, d\right)=D_{d}^{k}(w, \mu)\left(x^{i}, d\right) \\
\emptyset \quad & \text { if } P_{k}(w, \mu)\left(x^{i}, d\right) \neq D_{d}^{k}(w, \mu)\left(x^{i}, d\right)
\end{array}
$$

- Family of niono-valued realizations of optimal discrete controls associated to $(w, \mu), \mathrm{M}$

$$
\begin{aligned}
& M(w, \mu) \subset \Theta \\
& M(w, \mu)=\left\{A \in \Theta: A\left(x^{i}, d\right) \in \Phi(w, \mu)\left(x^{i}, d\right) ; \forall\left(x^{i}, d\right) \in V^{k} \times D\right\}
\end{aligned}
$$

Proposition 4.1 There exists at most one value of parameter $\mu^{k}$ such that (43) has a solution. $w \in F^{k}$.

Proof. Let $\left(w^{1}, \mu_{1}^{k}\right)$ and $\left(w^{2}, \mu_{2}^{k}\right)$ be two solutions of $(43)$, such that $\mu_{1}^{k}<\mu_{2}^{k}$.

For each $\left(w_{i}, \mu_{i}^{k}\right)$ we can take a policy $A^{i} \in \mathrm{M}\left(w_{i}, \mu_{i}^{k}\right)$.

Let $C=\left\{\left(x^{1}, d_{1}\right), \ldots,\left(x^{q}, d_{q}\right)\right\}$ be a cycle associated to $A^{2}$; for every $j=1, \ldots, q$, we have, by virtue of (43)

$$
w^{1}\left(x^{j}, d_{j}\right) \leq w^{1}\left(T\left(x^{j}, d_{j}\right), A^{2}\left(x^{j}, d_{j}\right)\right)+F_{d_{j}}\left(x^{j}, A^{2}\right)-\frac{\left(x^{j-1}\right)_{d_{j}}-\left(x^{j}\right)_{d_{j}}}{p_{d_{j}}-r_{d_{j}}} \mu_{1}^{k} .
$$

In consequence, by addition along the cycle we obtain

$$
\sum_{j=1}^{q} w^{1}\left(x^{j}, d_{j}\right) \leq \sum_{j=1}^{q}\left(w^{1}\left(T\left(x^{j}, d_{j}\right), A^{2}\left(x^{j}, d_{j}\right)\right)+F_{d_{j}}\left(x^{j}, A^{2}\right)-\frac{\left(x^{j+1}\right)_{d_{j}}-\left(x^{j}\right)_{d_{j}}}{p_{d_{j}}-r_{d_{j}}} \mu_{1}^{k}\right) .
$$


Taking into account Remark 4.5, we have

$$
\sum_{j=1}^{q} w^{1}\left(x^{j}, d_{j}\right) \leq \sum_{j=1}^{q} w^{1}\left(T\left(x^{j}, d_{j}\right), A^{2}\left(x^{j}, d_{j}\right)\right)
$$

therefore

$$
\sum_{j=1}^{q} F_{d_{j}}\left(x^{j}, A^{2}\right) \geq \sum_{j=1}^{q} \frac{\left(x^{j+1}\right)_{d_{j}}-\left(x^{j}\right)_{d_{j}}}{p_{d_{j}}-r_{d_{j}}} \mu_{1}^{k} .
$$

In consequence

$$
\mu_{1}^{k} \leq \frac{\sum_{j=1}^{q} F_{d_{j}}\left(x^{j}, A^{2}\right)}{\sum_{j=1}^{q} \frac{\left(x^{j+1}\right)_{d_{j}}-\left(x^{j}\right)_{d_{j}}}{p_{d_{j}}-r_{d_{j}}}} .
$$

In the same way we obtain

$$
\mu_{2}^{k}=\frac{\sum_{j=1}^{q} F_{d_{j}}\left(x^{j}, A^{2}\right)}{\sum_{j=1}^{q} \frac{\left(x^{j+1}\right)_{d_{j}}-\left(x^{j}\right)_{d_{j}}}{p_{d_{j}}-r_{d_{j}}}} .
$$

Therefore it results $\mu_{2}^{k} \geq \mu_{1}^{k}$, which is a contradiction. Then $\mu_{2}^{k}=\mu_{1}^{k}$.

Remark 4.7 If $(w, \mu)$ is a solution of $(43)$, then $w+c \cdot e$ is a solution $\forall c \in \Re$. being $e=(1, \ldots, 1) \in \Re^{(m-1) \times N}$.

\subsubsection{Definition of operator $P_{k}^{\lambda}$}

We repeat here the definition of operator $D_{d}^{\lambda, k}$, introduced in [2]

$$
\begin{aligned}
& \begin{array}{r}
\left(D_{d}^{\lambda k} w\right)\left(x^{i}\right)=\left(1-\lambda h^{d}\right) w\left(x^{i}+h^{d} g(d), d\right)+h^{d} f\left(x^{i}, d\right) \\
\forall x^{i} \in\left(V^{k} \bigcap C \gamma_{k, d}^{-} \bigcap C\left(\bigcup_{r \neq d} \gamma_{k, d}^{-}\right)\right) .
\end{array} \\
& \left(D_{d}^{k} w\right)\left(x^{i}\right)=+\infty \quad \forall x^{i} \in\left(\stackrel{-}{\gamma_{k, d}} \bigcup\left(\bigcup_{r \neq d} \gamma_{k, d}^{-}\right)\right) .
\end{aligned}
$$

The operator $P_{k}^{\lambda}: F_{k} \rightarrow F_{k}$ is defined by

$$
\left(P_{k} w\right)\left(x^{i}, d\right)=\min \left(\left(D_{d}^{k} w\right)\left(x^{i}\right),\left(S^{d}(w)\right)\left(x^{i}\right)\right), \quad \forall x^{i} \in V^{k}, \forall d \in D,
$$

and the following problem allows us to find the unique solution $U^{\lambda, k}$ of the discrete discounted cost problem

Problem $P_{k}^{\lambda}:$ Find the fixed point of operutor $P_{k}^{\lambda}$. 


\subsection{Relation between problems $\mathbf{P}_{k}$ and $\mathbf{P}_{k}^{\lambda}$}

The relation between these two problens is established in the following form.

Theorema 4.1 By virtue of feasibility condition (6), we have

$$
\lim _{\lambda \rightarrow 0} \lambda U^{\lambda, k}\left(x^{i}, d\right)=\mu^{k} \quad \forall x^{i} \in V^{k}, \forall d \in D .
$$

Also, $\forall x^{i_{0}} \in V^{k}, \forall d_{0} \in D$

$$
\forall w \in\left(\bigcap_{\varsigma>0}\left(\bigcup_{\varsigma>\lambda>0}\left(U^{\lambda, k}(\cdot)-U^{\lambda, k}\left(x^{i_{0}}, d_{0}\right) \cdot e\right)\right)\right), \quad\left(u, \mu^{k}\right) \text { is a solution of } P_{k}
$$

Proof. In first place let us see that for each pair $\left(x^{i}, d\right),\left\{\lambda U_{d}^{\lambda, k}\left(x^{i}, d\right): \lambda \in \Re^{-}\right\}$is bounded.

Let $\left(x^{i}, d\right) \in V^{k} \times D$, by virtue of the construction of the mesh, there exists a closed cycle to which it belongs; therefore, there exists $M(k)$ (which does not depend on $\lambda$ ) such that

$$
U^{\lambda, k}\left(x^{i}, d\right) \leq \frac{M(k)}{\lambda}
$$

then

$$
0 \leq \lambda U^{\lambda, k} \leq M(k)
$$

Moreover, two points $\left(x^{i}, d\right)$ and $\left(x^{i_{0}}, d_{0}\right)$ of $V^{k} \times D$, can be joined by a path. This path has an associated cost that is uniformuly-bounded with respect to $\lambda$. in consequence there exist.s $K\left(x^{i}, x^{i_{0}}, d, d_{0}\right)$ such that

$$
\left|U^{\lambda, k}\left(x^{i}, d\right)-U^{\lambda, k}\left(x^{i_{0}}, d_{0}\right)\right| \leq K\left(x^{i}, x^{i_{0}}, d, d_{0}\right) .
$$

Let $\left\{\lambda^{\nu}\right\}$ be a sub-sequence such that there exists $\tilde{\mu}, u\left(x^{i}, d\right)$ for which the following convergence holds.

$$
\begin{gathered}
\lambda^{\nu} U^{\lambda^{\nu}, k}\left(x^{i_{0}}, d_{0}\right) \rightarrow \tilde{\mu} \\
U^{\lambda, k}\left(x^{i}, d\right)-U^{\lambda, k}\left(x^{i_{0}}, d_{0}\right) \rightarrow w\left(x^{i}, d\right) .
\end{gathered}
$$

By virtue of (49), $\forall x^{i}$ and $\forall d$

$$
\lambda^{\nu} U^{\lambda^{\nu}, k}\left(x^{i_{0}}, d_{0}\right) \rightarrow \tilde{\mu} .
$$

Let us see that $(w, \tilde{\mu})$ is a solution of $\mathrm{P}^{k}$. We have, $\forall x^{i} \in V^{k}, \forall d=0, \ldots, m$,

$$
\begin{aligned}
& U^{\lambda^{\nu}, k}\left(x^{i}, d\right)-U^{\lambda^{\nu} ; k}\left(x^{i_{0}}, d_{0}\right)=\left(P_{k}^{\lambda} U^{\lambda^{\nu}, k}\right)\left(x^{i}, d\right)-U^{\lambda^{\nu}, k}\left(x^{i_{0}}, d_{0}\right)= \\
& \min \left(\left(D_{d}^{\lambda, k} U^{\lambda^{\nu}, k}\right)\left(x^{i}\right)-U^{\lambda^{\nu}, k}\left(x^{i_{0}}, d_{0}\right),\left(S^{d} U^{\lambda^{\nu}, k}\right)\left(x^{i}\right)-U^{\lambda^{\nu}, k}\left(x^{i_{0}}, d_{0}\right)\right) .
\end{aligned}
$$




$$
\begin{aligned}
& \left(D_{d}^{\lambda, k} U^{\lambda^{\nu}, k}\right)\left(x^{i}\right)-U^{\lambda^{\nu}, k}\left(x^{i_{0}}, d_{0}\right)= \\
& \quad U^{\lambda^{\nu}, k}\left(x^{i}+h^{d} g(d), d\right)-U^{\lambda^{\nu}, k}\left(x^{i_{0}}, d_{0}\right)+h^{d} f\left(x^{i}, d\right)-\lambda^{\nu} h^{d} U^{\lambda^{\nu}, k}\left(x^{i}+h^{d} g(d), d\right) .
\end{aligned}
$$

By taking limit when $\lambda^{\nu} \rightarrow 0$, we obtain

$$
\left(D_{d}^{k}(w, \tilde{\mu})\right)\left(x^{i}\right)=w\left(x^{i}+h^{d} g(d), d\right)+h^{d} \cdot\left(f\left(x^{i}, d\right)-\tilde{\mu}\right) .
$$

Moreover, as

$$
\left(S^{d} U^{\lambda^{\nu}, k}\right)\left(x^{i}\right)-U^{\lambda^{\nu}, k}\left(x^{i_{0}}, d_{0}\right)=\min _{\tilde{d} \neq d}\left(q(d, \tilde{d})+U^{\lambda^{\nu}, k}\left(x^{i}, \tilde{d}\right)-U^{\lambda^{\nu},{ }_{k}}\left(x^{i_{0}}, d_{0}\right)\right),
$$

we have

$$
\lim _{\lambda^{\nu} \rightarrow 0}\left(S^{l} U^{\lambda^{\nu}, k}\right)\left(x^{i}\right)-U^{\lambda^{\nu}, k}\left(x^{i_{0}}, d_{0}\right)=S^{d}(w)\left(x^{i}\right) .
$$

Finally, $\forall x^{i} \in V^{k}, \forall d=0, \ldots, m$, we have

$$
\begin{aligned}
& \lim _{\lambda^{\nu} \rightarrow 0} U^{\lambda^{\nu}, k}\left(x^{i}, d\right)-U^{\lambda^{\nu}, k}\left(x^{i_{0}}, d_{0}\right) \\
& \quad=\lim _{\lambda^{\nu} \rightarrow 0}\left(\min \left(\left(D_{d}^{\lambda, k} U^{\lambda^{\nu}, k}\right)\left(x^{i}\right)-U^{\lambda^{\nu}, k}\left(x^{i_{0}}, d_{0}\right),\left(S^{d} U^{\lambda^{\nu}, k}\right)\left(x^{i}\right)-U^{\lambda^{\nu}, k}\left(x^{i_{0}}, d_{0}\right)\right)\right) \\
& \quad=\min \left(\lim _{\lambda^{\nu} \rightarrow 0}\left(D_{d}^{\lambda, k} U^{\lambda^{\nu}, k}\right)\left(x^{i}\right)-U^{\lambda^{\nu}, k}\left(x^{i_{0}}, d_{0}\right), \lim _{\lambda^{\nu} \rightarrow 0}\left(S^{d} U^{\lambda^{\nu} k}\right)\left(x^{i}\right)-U^{\lambda^{\nu}, k}\left(x^{i_{0}}, d_{0}\right)\right) \\
& \quad=\min \left(\left(D_{d}^{k}(w, \tilde{\mu})\right)\left(x^{i}\right), S^{d}(w)\left(x^{i}\right)\right),
\end{aligned}
$$

i.e., we have

$$
w\left(x^{i}, d\right)=\min \left(\left(D_{d}^{k}(w, \tilde{\mu})\right)\left(x^{i}\right), S^{d}(w)\left(x^{i}\right)\right),
$$

in consequence $(w, \tilde{\mu})$ is a solution of $\mathrm{P}^{k}$. From Proposition $4.1 \mathrm{it}$ results

$$
\tilde{\mu}=\mu^{k} \text {. }
$$

As the subsequence $\lambda^{\nu}$ that we have chosen is arbitrary, we conclude that there exists a unique accumulation point. This conclusion implies that all the sequence is convergent, i.e.

$$
\lim _{\lambda \rightarrow 0} \lambda U^{\lambda, k}\left(x^{i}, d\right)=\mu^{k}
$$

Remark 4.8 In fact it can be proved - using basically the fact that the set of feedback policies of the discrete problem is finite - that the following estimation of the convergence velocity holds

$$
\left|\lambda U^{\lambda, k}-\mu^{k}\right| \leq C(k) \lambda .
$$

The proof uses elementary arguments and it will be omitted for the sake of briefness. 


\subsection{Convergence of the method}

Theorema 4.2 The following estimation for the difference betuecn the optimal average cost of the original continuous problem and those corresponding to its discrete appromimation holds

$$
\left|\mu-\mu^{k}\right| \leq C k
$$

Proof. We use the following triangular inequality

$$
\left|\mu-\mu^{k}\right| \leq\left|\mu-\lambda U^{\lambda}\right|+\lambda \| U^{\lambda}-U^{\lambda, k}||+\left|\lambda U^{\lambda, k}-\mu^{k}\right| .
$$

By taking limit when $\lambda \rightarrow 0$ we obtain (53), since by virtue of [2] it, is verified

$$
\left\|U^{\lambda}-U^{\lambda, k}\right\|_{K^{*}} \leq \frac{C\left(K^{-}\right)}{\lambda} k
$$

being

$$
\left\|U^{\lambda}-U^{\lambda, k}\right\|_{K^{-}}=\max \left\{\left|U_{d}^{\lambda}(x)-U^{\lambda, k}(x, d)\right|: x \in V^{k} \bigcap K, d \in D\right\},
$$

where $K$ is a compact set that does not depend on $k$ such that

$$
K \subset Q, K \cap \partial Q^{-}=\emptyset
$$

\section{Numerical algorithm}

We define here an algorithm which uses value iteration and policy iteration techniques and also makes use of the properties established in Theorem 3.2. This algorithm takes into account the methods described in [17], [21] and it is a natural modification of the algorithm presented in [2].

\subsection{Preliminary definitions}

- $\epsilon$-suboptimal multi-valued discrete controls associated to $w: A_{\epsilon}$

$$
\begin{aligned}
& A_{\epsilon} \in \Lambda \\
& \left(A_{\epsilon} w\right)\left(x^{i}, d\right)=\left(B_{\epsilon} w\right)\left(x^{i}, d\right) \cap\left(C_{\epsilon} w\right)\left(x^{i}, d\right),
\end{aligned}
$$

where

$$
\begin{aligned}
& \left(B_{c} w\right)\left(x^{i}, d\right)=\left\{\tilde{d} \in D:\left(P_{k} w\right)\left(x^{i}, d\right)+\epsilon \geq q(d, \tilde{d})+w\left(x^{i}, \tilde{d}\right)\right\} \\
& \left(C_{\epsilon} w\right)\left(x^{i}, d\right)=\mid \begin{array}{ccc}
\{d\} & \text { if } & \left(P_{k} w\right)\left(x^{i}, d\right) \geq\left(D_{d}^{k} w\right)\left(x^{i}\right)-\epsilon . \\
\emptyset & \text { if } & \left(P_{k} w\right)\left(x^{i}, d\right)<\left(D_{d}^{k} w\right)\left(x^{i}\right)-\epsilon
\end{array}
\end{aligned}
$$


- Linear system associated to a feedback discrete policy $\tilde{A} \in \Theta$.

We consider a linear system that we denote in a brief way

$$
L(u, \mu)=b .
$$

For this system, the relation that defines each equation is

$$
\mid \begin{array}{ll}
u\left(x^{i}, d\right)=\left(D_{d}^{k} u, \mu\right)\left(x^{i}\right) & \text { if } \quad \tilde{A}(u)\left(x^{i}, d\right)=d \\
u\left(x^{i}, d\right)=q(d, \tilde{d})+u\left(x^{i}, \widetilde{d}\right) & \text { if } \quad \tilde{A}(u)\left(x^{i}, d\right)=\tilde{d} .
\end{array}
$$

For the discount problem $\lambda$, we also consider the linear systeru associated to a policy $\tilde{A} \in \Theta$

$$
L^{\lambda} u=\beta,
$$

where the relation that defines each equation is

$$
\mid \begin{array}{ll}
u\left(x^{i}, d\right)=\left(D_{d}^{\lambda, k} u\right)\left(x^{i}\right) & \text { if } \quad \tilde{A}(u)\left(x^{i}, d\right)=d . \\
u\left(x^{i}, d\right)=q(d . \tilde{d})+u\left(x^{i}, \tilde{d}\right) & \text { if } \quad \tilde{A}(u)\left(x^{i} \cdot d\right)=\tilde{d} .
\end{array}
$$




\subsection{A fast algorithm}

Step 1: $\quad$ Set $\lambda>0,0<\gamma<1, w^{1,0} \in F_{k}, \bar{r}>0, \epsilon>0, \nu=0, \lambda^{\prime \prime}=\lambda$

Step 2: $\quad \nu=\nu+1, \eta=0, A^{\nu}=\{\emptyset\}^{(m+1) \times N}$

Step 3: $\quad \eta=\eta+1$, compute $w^{\nu, \eta}=P_{k}^{\lambda^{\nu}}\left(w^{\nu, \eta-1}\right), A^{\nu, \eta}=A_{\mathrm{\epsilon}}\left(w^{\nu, \eta}\right)$

Step 4: If $A^{\nu, \eta}=A^{\nu}, r=r+1$, and go to Step 5 else, $r=0, A^{\nu}=A^{\nu, \eta}$, and go to Step 3 .

Step 5: $\quad$ If $r \geq \bar{r}, \quad$ go to Step 3

else, choose any $\hat{A}^{\nu, \eta} \in \Theta$ such that $\forall\left(x^{i}, d\right), \hat{A}^{\nu, \eta}\left(x^{i}, d\right) \in A^{\nu, \eta}\left(x^{i}, d\right)$ and construct the system $L^{\lambda}$ associated to $\hat{A}^{\nu, \eta}$

Step 6: If $\operatorname{det}\left(L^{\lambda}\right) \neq 0$, compute the solution $v$ of the system $L^{\lambda} v=\beta$ else, $r=0, A^{\nu}=\{\emptyset\}^{(m+1) \times N}$, and go to Step 3 .

Step 7: If $v \neq P_{k}^{\lambda^{\nu}} v$, if $\left(\nu=0\right.$ or $\left.v \leq w^{\nu, \eta}\right), \quad w^{\nu+1,0}=v, \nu=\nu+1, \eta=0, r=0$, $A^{\nu}=\{\emptyset\}^{(m-1) \times N}$, and go to Step 3

Step 8: $\quad$ Form the system $L$ associated to $\hat{A}^{\nu, \eta}$

Step 9: Test if the system $L\left(u, \mu^{k}\right)$ has at least one solution and conpute it else, go to Step 11

Step 10: If $\operatorname{TEST}\left(u, \mu^{k}, \hat{A}^{\nu, \eta}\right)=1$, end $\left(\hat{A}^{\nu, \eta}\right.$ is an optimal policy)

Step 11: $\quad w^{\nu-1,0}=\frac{1}{\gamma} v, \lambda^{\nu+1}=\gamma \lambda^{\nu}$ and go to Step 2

Remark 5.1 Essentially the function TEST must check that the policy $\hat{A}^{\nu, \eta}$, is optimal for the discrete problem with average criterion. This property holds if the pair $\left(u, \mu^{k}\right)$ is a solution of (43).

Although the pair could not be a solution of (43), the policy $\hat{A}^{\nu, \eta}$ may be optimal in the sense that there is another pair $\left(w, \mu^{k}\right)$ which is a solution of the system $L\left(w, \mu^{k}\right)=b$, and that in addition it is a solution of (43). If this holds, TEST brings a function with that property. 


\subsubsection{Definition of the auxiliary function TEST}

The function $\operatorname{TEST}\left(u, \mu^{k}, \hat{A}\right)$ is computed by the following algorithn:

Step 1: $\quad v^{\prime}=1, w^{1}=u, A^{r}=\hat{A}$ compute $S^{\dot{c}}=\{$ cycles associated to $\hat{A}\}$

Step) 2: if $w^{v}=P_{k}\left(w^{v}, \mu^{k}\right)$ TEST $=1$. End.

Step 3: $\quad v=P_{k}\left(w^{v}, \mu^{k}\right)$ choose $\left(x^{0}, d_{0}\right)$, such that $v\left(x^{0}, d_{0}\right)<w^{v}\left(x^{0}, d_{0}\right)$

Step 4: compute $\Theta\left(w^{v}, \mu^{k}\right)$ and choose any $\tilde{A} \in \mathrm{M}\left(w^{v}, \mu^{k}\right)$ form $A \in \Theta$ in the following way:

$$
\begin{aligned}
& A\left(x^{i}, d\right)=A^{r}\left(x^{i}, d\right), \text { if }\left(x^{i}, d\right) \neq\left(x^{0}, d_{0}\right) \\
& A\left(x^{0}, d_{0}\right)=\tilde{A}\left(x^{0}, d_{0}\right)
\end{aligned}
$$

Step 5: compute $C=\{$ circles associated to $A\}$

if $C$ is not included in $\tilde{C}$, TEST $=0$. End

else, find the maxinum element of the set of vectors $w$ that

$$
\mid \begin{aligned}
& L\left(w, \mu^{k}\right)=b \\
& \max _{C} u \leq 0
\end{aligned}
$$

where $L$ is the linear system associated to $A$

$v=v+1, w^{v}=w, A^{r}=A$, and go to Step 2 .

Remark 5.2 The function TEST is defined in such a way that when for a pair $w$ - solution of the L system associated to policy $\hat{A}^{\nu, \eta}$ - the optinality condition is satisfied, we have

$$
\operatorname{TEST}\left(u, \mu^{k}, \hat{A}^{\nu, \eta}\right)=1 .
$$

Remark 5.3 It is clear that the maximum element computed in Step 5, can be found by solving a linear programming problem. The existence of maximum element is immediate and the search of this element can be solved by an ad-hoc algorithm withont using a general linear programming procedure.

Proposition 5.1 The algorithm, which computes the values of the TEST function, converges in a finite number of steps. 
Proof. Let us suppose, by reductio ad absurdum, that the algoritim originates an infinite sequence of values $w^{v}$. By construction, it would generate a sequence of Markov's chains where the sets of associated cycles are non-increasing in the following sense

$$
C\left(A^{v+1}\right) \subseteq C\left(A^{v}\right)
$$

In consequence, after a finite number of steps $C\left(A^{\bar{v}}\right)$ would remain fixei. From this condition it is possible to see that the sequence of functions $w^{v}$ is decreasing and can only take a finite number of values. This is a contradiction to the supposition that the sequence is infinite.

Lemma 5.1 If in the previous algorithm the set $S^{\hat{c}}$ is not included in. $S^{\dot{c}}$, there exists another cycle $C$ such that $\mu(C)<\mu^{k}$.

Proof. Let $C$ be a new cycle with $q$ nodes that, without losing generality, we will denote by

$$
\left.C=\left\{\left(x^{1}, d_{1}\right), \ldots, x^{q}, d_{q}\right)\right\}
$$

i.e.

$$
\begin{gathered}
\left(x^{j+1}, d_{j+1}\right)=\left(T\left(x^{j}, d_{j}\right), A\left(x^{j}, d_{j}\right)\right) \\
\left(x^{1}, d_{1}\right)=\left(T\left(x^{q}, d_{q}\right), A\left(x^{q}, d_{q}\right)\right)
\end{gathered}
$$

By construction, we have that for every $j=1, \ldots, q$ it is verified

$$
w\left(x^{j}, d_{j}\right) \geq w\left(T\left(x^{j}, d_{j}\right), A\left(x^{j}, d_{j}\right)\right)+F_{d_{j}}\left(x^{j}, x^{j-1}, A\left(x^{j}, d_{j}\right)\right)-\left(\frac{\left(x^{j-1}\right)_{d_{j}}-\left(x^{j}\right)_{d_{j}}}{p_{d_{j}}-r_{d_{j}}}\right) \mu^{k},
$$

where at least one inequality is strict, hence

$$
\begin{aligned}
& \sum_{j=1}^{q} w\left(x^{j}, d_{j}\right) \geq \\
& \quad \sum_{j=1}^{q}\left(w\left(T\left(x^{j}, d_{j}\right), A\left(x^{j}, d_{j}\right)\right)+F_{d_{j}}\left(x^{i}: x^{j-1}, A\left(x^{j}, d_{j}\right)\right)-\left(\frac{\left(x^{j-1}\right)_{d_{j}}-\left(x^{j}\right)_{d_{j}}}{p_{d_{j}}-r_{d_{j}}}\right) \mu^{k}\right) .
\end{aligned}
$$

As

$$
\sum_{j=1}^{q} w\left(x^{j}, d_{j}\right)=\sum_{j=1}^{q} w\left(T\left(x^{j}, d_{j}\right), A\left(x^{j}, d_{j}\right)\right)
$$

we obtain

$$
\sum_{j=1}^{q} F_{d_{j}}\left(x^{j}, x^{j-1}, A\left(x^{j}, d_{j}\right)\right)<\sum_{j=1}^{q}\left(\frac{\left(x^{j-1}\right)_{d_{j}}-\left(x^{j}\right)_{d_{j}}}{p_{d_{j}}-r_{d_{j}}}\right) \mu^{k}
$$


In consequence, the average $\tilde{\mu}^{k}$ along the new cycle satisfies

$$
\mu^{k}>\mu(C)=\frac{\sum_{j=1}^{q} F_{d_{j}}\left(x^{j}, x^{j: 1}, A\left(x^{j}, d_{j}\right)\right)}{\sum_{j=1}^{q}\left(\frac{\left(x^{j \div 1}\right)_{d_{j}}-\left(x^{j}\right)_{d_{j}}}{p_{d_{j}}-r_{d_{j}}}\right)} .
$$

\subsection{Convergence of the algorithm}

Theorema 5.1 The algorithm. converges in a finite number of steps

Proof. The algorithm cannot remain in an infinite loop from Step 3 to Step 7 by virtue of [13]. We will also see that the algorithm can not produce an infinite sequence by passing through Step 11. In effect, if it passed through Step 11 an intinite number of tines, it would generate a sequence $\lambda^{\nu} \rightarrow 0$.

In fact, for the $\tilde{A}^{\nu, \eta}$ obtained after Step 7 , we have that for every cycle associated to $C$,

$$
\lambda^{\nu} U^{\lambda^{\nu}, k}\left(x^{i}, d\right) \rightarrow \mu(C) \quad \forall\left(x^{i}, d\right) \in C .
$$

At the same time. by virtue of Theorem 4.1

$$
\lambda^{\nu} U^{\lambda^{\nu}, k}\left(x^{i}, d\right) \rightarrow \mu^{k} \quad \forall\left(x^{i}, d\right) \in V^{* k} \times D
$$

then, from some $\bar{\nu}$ it, results $\forall C$

$$
\mu(C)=\mu^{k},
$$

i.e. the policy $\tilde{A}^{\nu, \eta}$ : is optimal, then it results $\mathrm{TEST}=1$ and the algorithm finishes in a finite number of steps generating the optimal discrete solution. 


\section{Applications}

We have applied the above presented procedure to an example with $m=2$ items and a discretization of $Q$ which comprises $50 \times 50$ nodes. The instantaneous cost function is linear and does not depend on the parameter $d$, i.e.

$$
f\left(x_{1}, x_{2}\right)=C_{1} x_{1}+C_{2} x_{2} \quad \forall d \in D .
$$

In Figure 2 it is shown the optimal trajectory obtained.

\begin{tabular}{|l||l|}
\hline \hline$M_{1}=0.833$ & $M_{2}=0.833$ \\
\hline$r_{1}=1$ & $r_{2}=1$ \\
\hline$p_{1}=6$ & $p_{2}=1.5$ \\
\hline$C_{1}=0.1$ & $C_{2}=0.1$ \\
\hline$q_{0,1}=15$ & $q_{1,0}=0$ \\
\hline$q_{0,2}=3$ & $q_{2,0}=0$ \\
\hline$q_{2,1}=15$ & $q_{1,2}=3$ \\
\hline$h_{1}=0.017$ & \\
\hline
\end{tabular}

Table 1: 


\section{Conclusions}

In this paper, we have presented both analytic and numerical results concerning the optimization of the production schedule of a multi-item single machine system, for the case of infinite horizon and average cost criterion.

We have analyzed the associated HJB equation (which here takes the form of a variational inequalities system of H-J-B type). We have proved the existence of solution of this QVI system and the uniqueness of the optimal average cost.

We have also obtained a method of discretization which principal features is that the approximation has a $k$-order precision. This property stems from the fact that the optimal cost functions of the problems with actualization coefficients $\lambda$ are uniformly Lipschitz continuous with respect to the parameter $\lambda$, and from the especial type of mesh used in the triangulation procedure.

In addition, we have developed a computational algorithm that obtains the solution of the discrete problem in a finite number of steps. This property is also a consequence of the especial type of mesh used. 


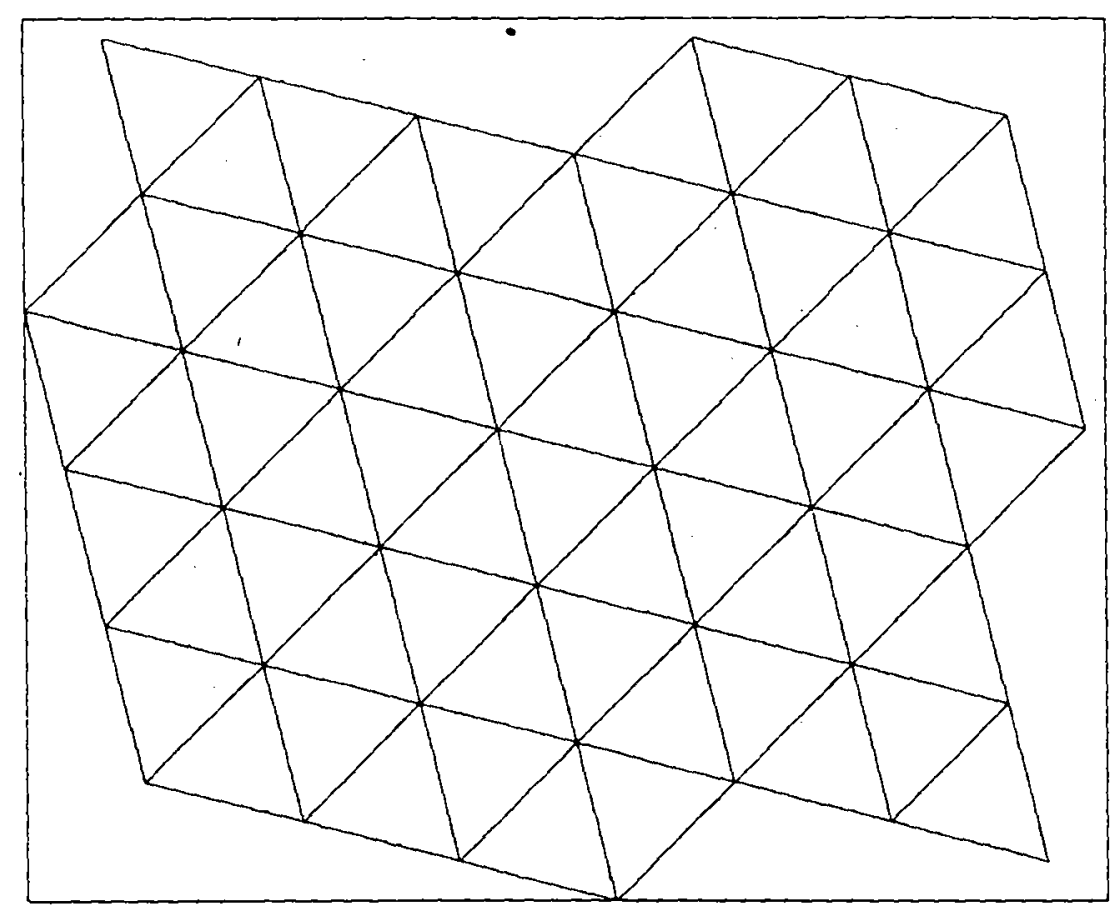

Figure 1: The mesh of $\Omega$ 


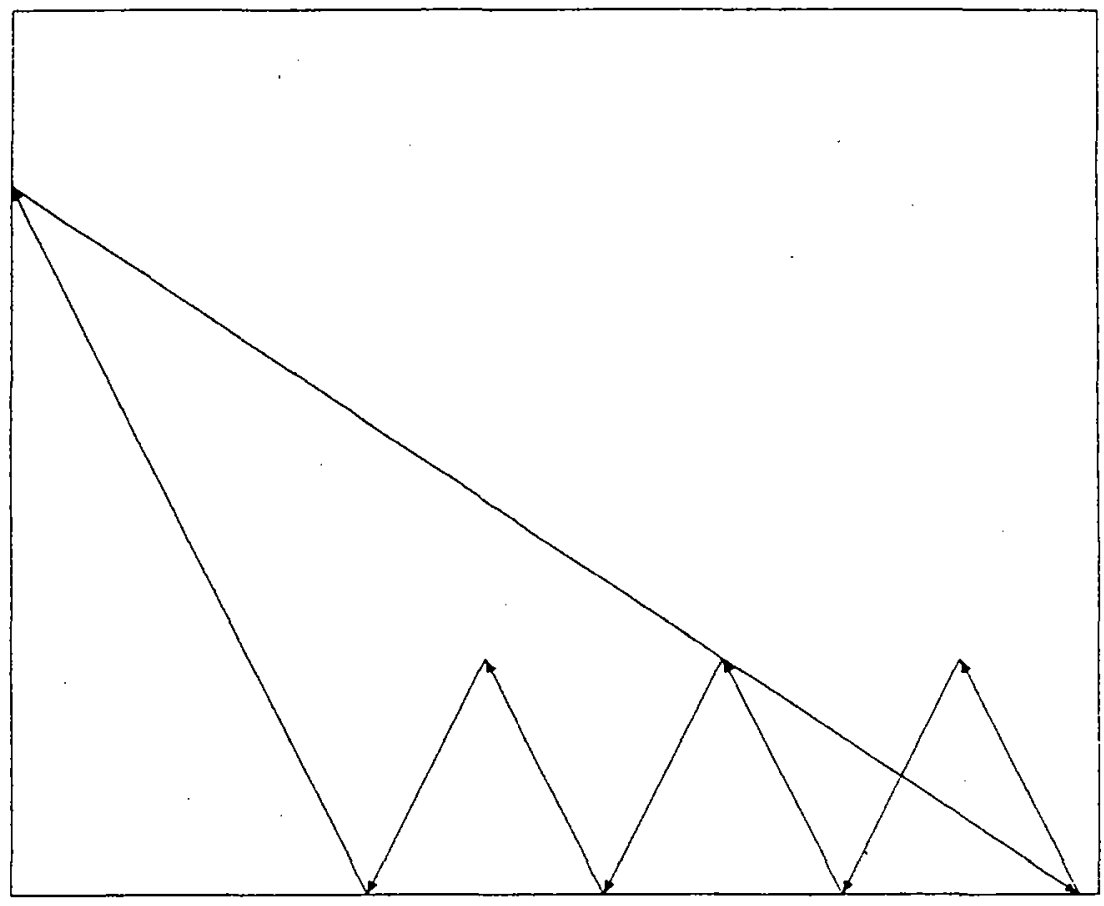

Figure 2: State space trajectory 


\section{References}

[1] Arapostathis A., Borkar V., Fernández E., Ghosh M., Marcus S., Discrete-time controlled Markon processes with anerage cost criterion: a surwey, SIAM J. Control Optim., Vol. 31, No2, pp. 282-344, 1993.

[2] Aragone L.S., González R.L.V., A fast computational procedure to solve the multi-item single machine lot scheduling optimization problem, Rapport de Recherche $\mathrm{N}^{\circ} 2616$. INRIA, 1995, (to appear in JOTA, 1996).

[3] Capuzzo Dolcetta 1., On a discrete approximation of the Hamilton-Jacobi equation of dynamic: programming, Journal of Applied Mathematie's and Optinization. Vol. 10. pp). 367-377, 1983.

[4] Capuzzo Dolcetta I., Evans L.C.. Optimal suitching for ordinary differential cquations. SIAM J. Control Optim., Vol. 22, 1984, pp. 143-161.

[5] Capuzzo Dolcetta I., Ishii H., Approximate solution of the Bellman equation of deterministic control theory, Journal of Applied Mathematics and Optimization. Vol. 11, pp. 161-181, 1984.

[6] Ciarlet P.G., Discrete maximum principle for finite-difference operators. Aequationes Mathematicae. Vol. 4, No3, pp. 338-352. 1970.

[7] Crandall M.G., Evans L.C.. Lions P.-L., Some properties of viscosity solutions of Hamilton-Jacobi equations, Trans. Amer. Math. Soc.. Vol. 282. pp. 487-502, 1984.

[8] Federgruen A., Schweitzer P.J., A survey of asymptotic: value-iteration for undiscounted Markovian decision processes, Recent developments in Markov decision processes, Hartley R., Thomas L. C., White D. J., Eds., Academic: Press, London. pp. 73-109, 1980.

[9] Fleming W.H., Rishel R.W., Deterministic and stochastic optimal control, SpringerVerlag, New York, 1975.

[10] Glowinski R., Numerical methods for nonlinear variational problems, Springer-Verlag, New York, 1984.

[11] Glowinski R., Lions J.L., Tréruolières R., Numerical analysis of variational inequalities, North Holland, Anisterdanı, 1981.

[12] González R.L.V., Muramatsu K., Rofman E., Quasi-variational inequality approach to multi-item single machine lot scheduling problem, in System Modeling and Optimization, Lecture Notes in Control and Information Sciences Vol. 180, pp. 885-893, Springer Verlag, New York, 1992. 
[13] Gonzálız R.L.V., Muramatsu K., Rofman E., Quasi-nariational incequality approach. to multi-item single machine lot scheduling problem, Rapport. de Recherche $\mathrm{N}^{\circ} 2057$. INRIA, 1993.

[14] González, R.L.V., Rofman E., On deterministic control problems: an approximation procedure for the optimal cost, Parts I-II, SIAM J. Control Optim., Vol. 23, N², pp. $242-285,1985$.

[15] González R.L.V.. Rofman E., Sur des solutions non bornées de l'équation de Bellman associée aux problèmes de commutation optimale avec contraints sur l'etât, Comptes Rendus Acad. Sc. Paris, Serie I. Tome 316, pp. 1193-1198. 1993.

[16] González R.L.V.. Rofnan E.. On unbounded solutions of Bellman's equation associated to optimal switching control problems with state constraints, Journal of Applied Mathematies and Optinization, Vol. 31, pp. 1-17, 1995.

[17] González R.L.V., Sagastizábal C.A.. Un algorithme pour la résolution rapide d'équations discrètes de Hamilton-Jacobi-Bellman. Comptes Rendus Acad. Si. Paris, Serie I. Tome 311. pp. 45-50. 1990.

[18] González R.L.V.. Tidball M.M., Sur l'ordre de convergence des solutions discrétisées en. temps et en espace de l'équation de Hamilton-Jacobi. Comptes Rendus Acad. Sc. Paris. Serie I, Tone 314, pp. 479-482, 1992.

[19] González R.L.V.. Tidball M.M., On a discrete time approximation of the HamiltonJacobi equation of dynamic programming. Rapport de Recherche N $^{\circ} 1375$. INRIA. 1990.

[20] Gonzálex R.L.V., Tidball M.M.. On the rate of convergence of fully discrete solution.s of Hamilton-Jacobi Equations, Rapport de Recherche N¹376. INRIA, 1991.

[21] González R.L.V., Tidball M.M., Fast solution of general nonlinear fixed point problems. In System Modeling and Optimization, Lecture Notes in Control and Information Sciences Vol. 180, pp. 35-44, Springer Verlag; New York, 1992.

[22] Hartley R.. Thomas L. C., White D. J., Recent Developments in Markon Decision Processes, Academic Press, London. 1980.

[23] Kushner H.J., Dupuis F., Numerical methods for stochastic control problems in continuous time. Springer-Verlag, New York. 1992.

[24] Lions P.-L., Perthame B., Quasi variational inequalities and ergodic impulse control, SIAM .J. Control Optim., Vol. 24, N4, pp. 604-615, 1986.

[25] Menaldi J.L., Perthame B., Robin M., Ergodic problem for optimal stochastic surtching, Joirnal of Math. Analysis and Applitations, Vol. 147, N². 1990. 
[26] Menaldi .J.L., Robin M., Some singular control problems with long term average criterion, in Lecture Notes in Control and Inf. Si.i., pp. 424-432, Springer Verlag, New York, 1984.

[27] Robin M.. On some inpulse control problems with long run average cost. SIAM J. Control Optim., Vol. 19, N³, pp. 333-358, 1981.

[28] Robin M.. Long run average control of continuous time Markon processes: A survey. Act.a Appl. Math., Vol. 1, pp. 281-299, 1983.

[29] Schäl M.. Average optimality in dynamic programming with general state space. Math. of Operations Research. Vol. 18, No1, pp. 163-172. 1993. 
Unité de recherche INRIA Rocquencourt

Domaine de Voluceau - Rocquencourt - B.P. 105 - 78153 Le Chesnay Cedex (France)

Unité de recherche INRIA Lorraine - Technopôle de Nancy-Brabois-Campus scientifique 615, rue du Jardin Botanique - B.P. I01-54602 Villers lès Vancy Cedex (France)

Unité de recherche INRIA Rennes - IRISA. Campus universitaire de Beaulieu 35042 Rennes Cedex (France)

(inité de recherche INRIA Rhônc-Alpes 46, avenue Fúlix Viallet - 38031 Grenoble Cedex I (France)

Unité de recherche INRIA Sophia Antipolis - 2004, route des Lucioles - B.P. 93 - 06902 Sophia Antipolis Cedex (France)

Éditcur

INRIA - Domaine de Voluceau - Rocquencourt - B.P. 105 - 78153 Le Chesnay Cedex (France)

ISSN $0249-6399$ 